\title{
GRANTEE INVENTIONS
}

\section{Linear Displacement Transducer}

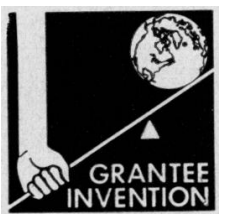

The linear displacement transducer is an electromechanical device that will indicate with a relative electrical output the position of a point in reference to a fixed point which is in line with the axis of the device. It is capable of measuring point excursions from 0 to $6.5 \mathrm{~cm}$. with a linearity deviation of \pm 0.5 percent of full scale.

The device consists of a polystyrene tube 6 inches long with a $3 / 8$-inch outside diameter and a $1 / 4$-inch inside diameter. Starting $1 / 4$ inch from each end, two single layer coils of No. 26 enamel-covered wire are wound in such a way that there is a 1-wire diameter space between each turn. Each coil is $31 / 8$ inches long and overlaps $3 / 4$ inch at the center. The windings are bifilar (side-by-side) in this area. A ferrite armature, two ferrite tubes cemented together (Ferroxcube Corp. of America No. 56$062-42 / 3 \mathrm{~A}$ ), and cemented to a fiberglass sensing rod, moves through the axis of the tube.

As the sensing rod is moved through its full excursion, 0 to $6.5 \mathrm{~cm}$., the armature will pass out of one coil, lowering its relative impedance, and into the other, raising its relative impedance. The extreme ends of the coils are connected across a high-frequency voltage source (one V 450KC). The ends at the overlap area are connected together. The output is taken from across either coil. This forms a variable voltage divider, the impedances of which are controlled by the position of the sensing rod.

The impedances of the individual coils do not change linearly with position. A common nonlinear element, deliberately induced by overlapping the coils, is canceled out by the effect of the voltage divider. All "simple" variable coils of this general type have a nonlinear relationship that cannot be canceled out in a voltage divider. There, the coils must be made linear or a controlled nonlinearity must be introduced.

The use of a high-frequency voltage source is made necessary by the low values of inductance involved. The a.c. output variations are converted to d.c. variations by a simple diode detector and r.c. filter.-J. French and A. A. Strbens, M.D., Rehabilitation Center, University of Wisconsin Medical Center, Madison. This invention was developed under Public Health Service research grant No. HE-04036.

\section{Sequential Roto-Disk Pollen Sampler}

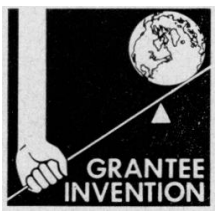

The sequential roto-disk pollen sampler is an impaction type device for use in collecting airborne ragweed pollen. It features a new method of automatic advancement of the collecting surface to provide sequential samples according to a preset schedule.

The momentum of particulates, large and heavy relative to air molecules, causes them to lag behind the airflow around an obstacle and to collide with it. With a suitable adhesive on the obstacle, the particulates are retained for microscopic studies.

The obstacle is the edge of an aluminum disk, 4.00 inches in diameter and 0.0937 inch thick. Two of these disks are mounted coplanar with a supporting plate with axes displaced from the center of the plate. The plate, at its center, is attached normal to a revolving shaft which permits impaction sampling because of the relative motion between the edge of the disk and the atmosphere. Discrete sampling surfaces on the edge of each disk are delineated by $3^{\circ}$ slots cut into the surrounding shields while a thin coating of silicone grease 